\title{
ELEVATED SERUM VASCULAR ENDOTHELIAL GROWTH FACTOR AND DECREASED SURVIVAL IN ADVANCED LARYNGEAL CARCINOMA
}

\author{
Theodoros N. Teknos, MD, ${ }^{1}$ Claudell Cox, MD, ${ }^{1}$ Sirius Yoo, BS, ${ }^{1}$ \\ Douglas B. Chepeha, MD, ${ }^{1}$ Gregory T. Wolf, MD, ${ }^{1}$ Carol R. Bradford, MD, ${ }^{1}$ \\ Thomas E. Carey, PhD, ${ }^{1}$ Susan G. Fisher, PhD $^{2}$ \\ ${ }^{1}$ Department of Otolaryngology-Head and Neck Surgery, University of Michigan Health System, 1500 E. \\ Medical Center Dr., Ann Arbor, Michigan 48109-0312. E-mail: teknos@umich.edu \\ ${ }^{2}$ Department of Biostatistics and Preventive Medicine, Loyola University, Cardinal Bernardin Cancer Center, \\ Maywood, Illinois
}

Accepted 17 May 2002

Published online 23 August 2002 in Wiley InterScience (www.interscience.wiley.com). DOI: 10.1002/hed.10163

\begin{abstract}
Purpose. The purpose of this study was to determine whether serum vascular endothelial growth factor (s-VEGF) levels at the time of diagnosis correlate with any known tumor variables and overall survival in patients with advanced laryngeal squamous cell carcinoma. Comparisons with a cohort of normal healthy controls were also performed to determine the potential usefulness of s-VEGF as a screening tool.

Experimental Design. Serum from patients enrolled in the VA Laryngeal Cooperative Study \#258 $(n=183)$, as well as normal healthy controls $(n=40)$ was used in this analysis. Quantitative enzyme-linked immunosorbent assays (ELISA) for VEGF were performed in duplicate on each serum sample. Demographic and survival data were available for each patient enrolled in the study. Univariate analyses, multivariate Cox regression analyses, and Kaplan-Meier survival analysis were used.

Results. The mean serum concentration of s-VEGF for the healthy control group was $47.83 \pm 0.13 \mathrm{pg} / \mathrm{mL}$. For all patients enrolled in the VA Cooperative Study, regardless of treatment group, the mean s-VEGF level was $317.22 \pm 25.46 \mathrm{pg} / \mathrm{mL}$. The patients randomly assigned to the surgical arm $(n=97)$ had a mean value of $315.44 \pm 30.44 \mathrm{pg} / \mathrm{mL}$. Those randomly assigned
\end{abstract}

Correspondence to: T. N. Teknos

(C) 2002 Wiley Periodicals, Inc. to the induction chemotherapy arm $(n=86)$ had a mean s-VEGF level of $319.22 \pm 42.11 \mathrm{pg} / \mathrm{mL}$. Serum VEGF levels were significantly elevated in patients with laryngeal carcinoma compared with healthy controls $(p<.001)$. The serum VEGF levels in each arm of the trial were also elevated versus the healthy controls ( $p$ $<.001$, surgery arm plus radiotherapy; $p<.001$, chemotherapy plus radiotherapy). In a univariate analysis, elevated s-VEGF correlated with poor Karnofsky performance status for all patients with advanced laryngeal carcinoma $(p<.008)$. High s-VEGF levels also correlated with a poor performance score in patients on the chemotherapy arm of the VA Laryngeal Trial $(p<.004)$. Elevated s-VEGF levels in the surgical plus radiotherapy arm correlated with node-positive disease $(p=.047)$ and supraglottic location of the tumor $(p=.022)$. In a multivariate analysis using all known tumor variables and s-VEGF levels, elevated s-VEGF levels and infiltrating growth pattern correlated with decreased survival for all evaluated patients with advanced laryngeal carcinoma ( $p=.065$, and $p=.018$, respectively).

Conclusions. Serum VEGF levels are significantly elevated in patients with advanced laryngeal carcinoma versus healthy controls. Elevated pretreatment s-VEGF levels tended to indicate a more aggressive disease state and a poorer overall survival in advanced laryngeal carcinoma. (C) 2002 Wiley Periodicals, Inc. Head Neck 24: 1004-1011, 2002

Keywords: angiogenesis; VEGF; head and neck cancer; organ preservation; serum marker 
Angiogenesis is the process of new blood vessel formation. It is encountered in essential physiologic processes (eg, endometrial proliferation, embryogenesis, wound healing), as well as in multiple pathologic conditions (eg, rheumatoid arthritis, diabetic retinopathy, neoplastic diseases). ${ }^{1}$ Folkman and coworkers ${ }^{2}$ pioneered the concept that primary or metastatic tumors must become vascularized to exceed $2 \mathrm{~mm}$. On the basis of animal data, Folkman et $\mathrm{al}^{3}$ found that tumor angiogenesis must precede tumor growth and that tumor progression can only occur when neoplasms switch from a prevascular to an angiogenic phase. Vascular endothelial growth factor (VEGF) is one of the most potent angiogenic factors discovered to date. Also known as vascular permeability factor (VPF), VEGF stimulates angiogenesis by increasing vascular permeability and by acting as an endothelial cell mitogen. ${ }^{4}$ Several studies have reported that elevated levels of serum VEGF (sVEGF) portend a poor prognosis in a variety of solid tumors. ${ }^{5-10}$ Furthermore, Qian et al ${ }^{11}$ have linked s-VEGF levels to metastatic disease in nasopharyngeal carcinoma. However, data related to other head and neck sites are lacking.

The purpose of this study was to determine whether s-VEGF levels, at the time of diagnosis, correlate with response to treatment and overall survival in patients with locally advanced laryngeal cancer. Comparisons with a cohort of normal healthy controls were also performed to determine the potential usefulness of s-VEGF as a screening tool.

\section{MATERIALS AND METHODS}

Patient Population. A total of 332 patients with stage III (188 patients) or stage IV (144 patients) squamous cell carcinoma of the larynx were enrolled in the prospective clinical trial entitled "The Department of Veteran's Affairs Cooperative Studies Laryngeal Cancer Study \#268."12 This cooperative study was reviewed and approved by the institutional review boards of all participating institutions. Informed consent was obtained from each patient at the time of accrual. Half of the patients enrolled in the study (166 patients) were randomly assigned to receive conventional surgery and postoperative radiation therapy (50-65 cGy). The other half (166 patients) received three cycles of induction chemotherapy (cis-platinum, $100 \mathrm{mg} / \mathrm{m}^{2}$ on day 1 and 5-fluorouracil, $1000 \mathrm{mg} / \mathrm{m}^{2}$ per day, on days 1 through 5) and radiation therapy (66-76 cGy). Surgical salvage was performed on those patients who failed to respond after two cycles of chemotherapy or on those with persistent or recurrent disease at the completion of treatment. This treatment regimen and study results have previously been described in detail. ${ }^{12}$ The sera used in this study were collected from each patient before treatment. It was centrifuged at $3000 \times g$ for $15 \mathrm{~min}$ and frozen at $-80^{\circ} \mathrm{F}$ until analysis. Ninety-seven samples from individual patients randomly assigned to the surgical arm of treatment and 86 samples from patients randomly assigned to treatment with chemotherapy were available for analysis. Forty serum samples from normal healthy volunteers were used as controls.

Serum VEGF Immunoassay. A quantitative, enzyme-linked immunosorbent assay (ELISA) was performed in duplicate on each sample to measure the concentration of soluble serum VEGF. A commercially available ELISA kit was used (Quantikine Human VEGF Immunoassay, R\&D Systems, Minneapolis MN). Serum samples from all patients were incubated for 2 hours at room temperature in duplicate $(100 \mu \mathrm{L})$ on microtiter plates coated with a monoclonal antibody specific for VEGF. Next, any unbound substances were washed away, and an enzyme-linked polyclonal antibody specific for VEGF was introduced. This was allowed to incubate for 2 hours at room temperature, and the plates were washed to remove unbound antibody. A substrate solution was added, and color development was stopped after 25 minutes at room temperature. A microplate reader was then used to determine colorimetric densities at $570 \mathrm{~nm}$ and $450 \mathrm{~nm}$ for each sample. The optical density for each sample was determined by subtracting the readings at $570 \mathrm{~nm}$ from the reading at $450 \mathrm{~nm}$. Results were calculated from a standard curve generated by a form parametric logistic curve fit and expressed in pg/ $\mathrm{mL}$ of serum. The test sensitivity as determined by the manufacturer is $<9.0 \mathrm{pg} / \mathrm{mL}$.

Statistical Analysis. All values are presented as mean \pm standard error. Student's $t$ test was used to detect differences between groups in continuous measures. Serum VEGF levels per specimen were compared between normal, healthy volunteers and laryngeal cancer patients using Student's $t$ test. Student's $t$ test was also used to determine whether there was a significant difference in s-VEGF levels among different categorical variables including clinical stages, $\mathrm{T}$ stages, $\mathrm{N}$ stages, Karnofsky performance scale scores, ages, 
growth patterns, treatment groups, and tumor sites. Survival analyses were conducted using the Kaplan-Meier technique; differences between distributions were compared using the log-rank test. Survival was calculated for each patient from the VA Larynx study randomization date to the date of death; patients remaining alive were censored at the date last known to be alive. A multivariate Cox regression model was used, and the number of covariates included s-VEGF ( $\mathrm{pg} / \mathrm{mL})$, growth pattern ( 1 or 2 vs 3 or 4 as described by Jakobsson ${ }^{13}$ ), treatment group (chemo/XRT vs surgery/ RT), stage (1-3 vs 4), site (glottic vs supraglottic), age, and $\mathrm{T}$ stage (1-3 vs 4$)$. For all analyses, a two-sided alpha level of .05 was considered statistically significant.

\section{RESULTS}

Patient Descriptive Data. There were a total of 183 patients with serum samples available for analysis from the 332 patients enrolled in the VA Laryngeal Cooperative Study. Ninety-seven were from the surgery arm and eighty-six were from the chemotherapy arm of the trial. For inclusive data on all the subjects refer to Table 1.

Most patients (112 of 183) had stage III tumors. The most common T stage was T3 (125 of $183)$, and the most common nodal status was N0 (105 of 183). Most tumors were supraglottic (116 of 183). Accurately reflecting the VA population, most patients were men (179 of 183) and white (142 of 183).

Serum VEGF Levels in Normal Controls vs Laryngeal Cancer Patients. The mean serum concentration of s-VEGF for the healthy control group was 47.83 $\pm 0.13 \mathrm{pg} / \mathrm{mL}$. For all patients in the VA Cooperative Study, regardless of treatment group, the mean s-VEGF level was $317.22 \pm 25.46 \mathrm{pg} / \mathrm{mL}$. The patients randomly assigned to the surgery arm (97 patients) had a mean value of $315.44 \pm$ $30.44 \mathrm{pg} / \mathrm{mL}$. Those randomly assigned to the induction chemotherapy arm (86 patients) had a mean s-VEGF level of $319.22 \pm 42.11$. Serum VEGF levels were significantly elevated in patients with laryngeal carcinoma compared with healthy controls $(p<.001)$. Also, when analyzed by treatment arm, each group had significantly elevated s-VEGF compared with the healthy controls (surgery plus RT, $p<.001$; induction chemotherapy plus RT, $p<.001)$. When comparing the s-VEGF levels in the two arms of the VA Laryngeal Study, there was no significant difference in s-VEGF levels ( $p=.941)$, as expected.

\begin{tabular}{|c|c|c|c|}
\hline & Surgery arm & Chemo arm & Total \\
\hline \multicolumn{4}{|l|}{ Stage } \\
\hline III & 59 & 53 & 112 \\
\hline IV & 38 & 33 & 71 \\
\hline \multicolumn{4}{|l|}{ T stage } \\
\hline 1 & 3 & 2 & 5 \\
\hline 2 & 7 & 4 & 11 \\
\hline 3 & 64 & 61 & 125 \\
\hline 4 & 23 & 19 & 42 \\
\hline \multicolumn{4}{|l|}{$\mathrm{N}$ stage } \\
\hline 0 & 58 & 47 & 105 \\
\hline 1 & 15 & 17 & 32 \\
\hline 2 & 14 & 10 & 24 \\
\hline 3 & 10 & 12 & 22 \\
\hline \multicolumn{4}{|l|}{ Tumor site } \\
\hline Glottic & 35 & 31 & 66 \\
\hline Supraglottic & 61 & 55 & 116 \\
\hline Subglottic & 1 & 0 & 1 \\
\hline \multicolumn{4}{|c|}{ Performance status } \\
\hline 60 & 4 & 5 & 9 \\
\hline 70 & 16 & 16 & 32 \\
\hline 80 & 30 & 19 & 49 \\
\hline 90 & 44 & 36 & 80 \\
\hline 100 & 3 & 10 & 13 \\
\hline \multicolumn{4}{|l|}{ Sex } \\
\hline M & 95 & 84 & 179 \\
\hline $\mathrm{F}$ & 2 & 2 & 4 \\
\hline \multicolumn{4}{|l|}{ Race } \\
\hline White & 83 & 59 & 142 \\
\hline Black & 13 & 25 & 38 \\
\hline Other & 1 & 2 & 3 \\
\hline \multicolumn{4}{|c|}{ Tumor growth pattern } \\
\hline 1 & 0 & 3 & 3 \\
\hline 2 & 55 & 45 & 100 \\
\hline 3 & 14 & 24 & 38 \\
\hline 4 & 4 & 3 & 7 \\
\hline
\end{tabular}

Correlation of S-VEGF Levels with Known Tumor Factors. Serum VEGF levels were compared in relation to a variety of known tumor prognostic variables. These variables included stage, $\mathrm{T}$ stage, $\mathrm{N}$ stage, site, race, growth pattern, and Karnofsky performance scale. Table 2 summarizes the results. When s-VEGF levels from patients in both arms of the trial are included in the analysis, the only factor that s-VEGF was significantly correlated with was Karnofsky Performance Scale ( $p=.008)$. High s-VEGF levels correlated with performance scores less than 80 , whereas levels below the mean correlated with performance scores greater than 80 . In patients randomly assigned to the induction chemotherapy arm, the s-VEGF levels were also significantly different between high and low performance status groups $(p=.004)$, but no other variables reached statistical significance. On the 
Table 2. Univariate analyses comparing serum VEGF levels with known tumor variables.

\begin{tabular}{|c|c|c|c|c|c|c|}
\hline & \multicolumn{2}{|c|}{ All patients } & \multicolumn{2}{|c|}{ Surgery arm } & \multicolumn{2}{|c|}{ Induction chemo arm } \\
\hline & Serum VEGF levels & $p$ Value & Serum VEFG levels & $p$ Value & Serum VEGF levels & $p$ Value \\
\hline \multicolumn{7}{|l|}{ Karnofsky PS } \\
\hline$\leq 80$ & $386.18(n=90)$ & * & $331.43(n=50)$ & & $454.62(n=40)$ & * \\
\hline$>80$ & $250.48(n=95)$ & .008 & $329.44(n=47)$ & .591 & $201.47(n=46)$ & .004 \\
\hline \multicolumn{7}{|l|}{ Growth pattern } \\
\hline 1 or 2 & $314.80(n=103)$ & & $293.90(n=55)$ & & $338.80(n=48)$ & \\
\hline 3 or 4 & $316.64(n=45)$ & .977 & $333.80(n=18)$ & .605 & $305.20(n=27)$ & .736 \\
\hline \multicolumn{7}{|l|}{ Stage } \\
\hline 3 & $297.71(n=112)$ & & $287.11(n=59)$ & & $296.83(n=53)$ & \\
\hline 4 & $357.45(n=71)$ & .209 & $359.43(n=38)$ & 248 & $355.17(n=33)$ & .504 \\
\hline \multicolumn{7}{|l|}{ Tumor stage } \\
\hline $\mathrm{T} 1-3$ & $307.09(n=141)$ & & $325.59(n=74)$ & & $286.65(n=67)$ & \\
\hline $\mathrm{T} 4$ & $351.22(n=42)$ & .468 & $382.79(n=23)$ & .552 & $434.07(n=19)$ & .147 \\
\hline \multicolumn{7}{|l|}{ Node stage } \\
\hline NO & $306.23(n=105)$ & & $261.61(n=58)$ & * & $361.30(n=47)$ & \\
\hline N1-3 & $332.00(n=78)$ & .618 & $395.51(n=39)$ & .047 & $268.49(n=39)$ & .267 \\
\hline \multicolumn{7}{|l|}{ Site } \\
\hline Supra/subglottic & $340.12(n=117)$ & & $361.81(n=62)$ & * & $315.68(n=55)$ & \\
\hline Glottic & $276.61(n=66)$ & .232 & $233.30(n=35)$ & .022 & $325.50(n=31)$ & .812 \\
\hline \multicolumn{7}{|l|}{ Race } \\
\hline Caucasian & $296.75(n=142)$ & & $300.34(n=83)$ & & $271.70(n=59)$ & \\
\hline All others & $388.10(n=41)$ & .258 & $404.98(n=14)$ & .225 & $379.35(n=27)$ & .337 \\
\hline
\end{tabular}

${ }^{*}$ Statistical significance.

other hand, in patients randomly assigned to the surgical arm, s-VEGF levels were lower in N0 vs $\mathrm{N}+$ patients $(p=.047)$ and between those patients with glottic tumors vs those with supraglottic tumors $(p=.022)$. No other variables, including performance scores, reached significance in the surgical arm patients.

Correlation of Serum VEGF Levels with Overall Survival in Patients with Advanced Laryngeal Cancer. Serum concentration of VEGF lacks a clearly defined cutoff level. It was determined a priori that differences in patient survival would be based on a comparison of subjects having an $\mathrm{s}$-VEGF level of greater than the sample mean plus one standard deviation versus all other subjects. Using this cutoff, elevated s-VEGF was significantly associated with reduced survival in the surgical arm of the VA Larynx Study $(p=.018)$ but not in the chemotherapy arm $(p=.90)$, as shown in the Kaplan-Meier survival curve analysis (see Figure 1). In a Cox regression model using VEGF as a continuous variable, once again elevated s-VEGF was associated with reduced survival in the surgical arm $(p=.0311)$ but not in the chemotherapy $\operatorname{arm}(p=.919)$. In a multivariate Cox regression model for overall survival, using s-VEGF levels from both arms of the trial and all the known tumor variables as covariates, s-
VEGF nearly obtained statistical significance ( $p$ $=.065$, see Table 3 ). Impressively, it did have the highest relative risk, imparting a $47 \%$ increased risk of death. The only variable significantly associated with reduced survival was aggressive growth pattern, and it imparted a $44 \%$ increased risk of death. When performing multivariate analysis in each arm of the trial individually using the same covariates, no variables were found to be correlated with poor overall survival in the chemotherapy arm (Tables 4 and 5). In the surgery arm, aggressive growth pattern correlated with poor survival ( $p=.011$ ); however, no other variables reached significance, including s-VEGF (Table 4).

\section{DISCUSSION}

VEGF is one of the most potent factors responsible for inducing tumor angiogenesis. ${ }^{4}$ It is a dimeric glycoprotein with at least six spliced variants containing 115, 121, 145, 165, and 206 amino acid residues. ${ }^{14-16}$ Each variant expresses identical biologic activity by binding to specific class III tyrosine receptor kinases (flt-1 and KDR). ${ }^{14-16}$ VEGF is expressed in both normal and neoplastic cells, where it acts as an endothelial cell mitogen. VEGF mRNA has been found to be overexpressed in tumor cells, and secreted VEGF has been localized to the supplying vascular endothelium of tu- 


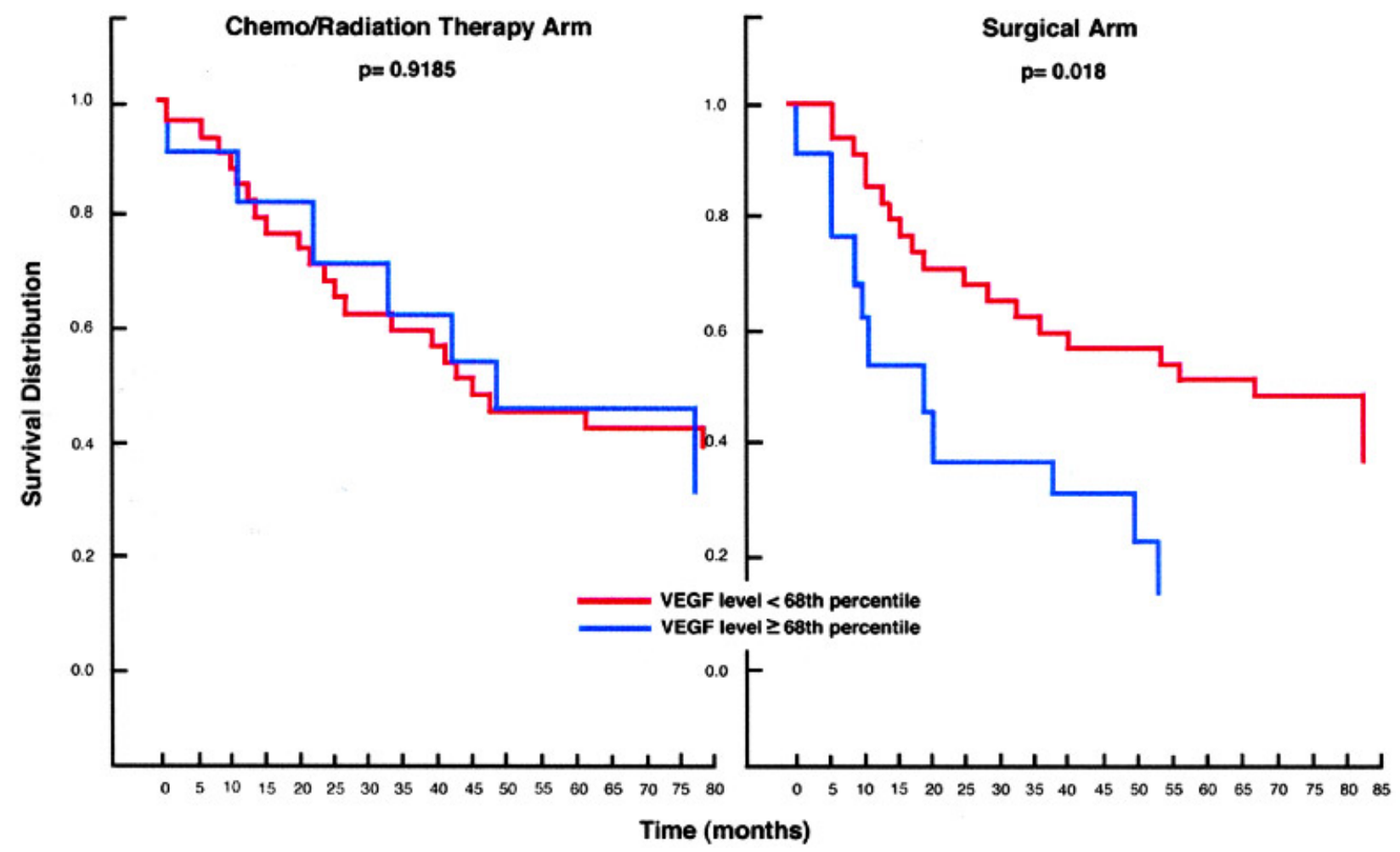

FIGURE 1. Kaplan-Meier curve survival analyses for the two arms of the VA laryngeal cooperative study \#268. Based on VEGF level there was no significant difference in survival distributions in the induction chemotherapy and radiation therapy arm. There was, however, a highly statistically significant difference between patients with a low level of angiogenesis versus those with a high level of angiogenesis in the surgical arm. [Color figure can be viewed in the online issue, which is available at www.interscience.wiley.com.]

mor cells. ${ }^{17}$ Deficiencies in critical nutrients, metabolites, and oxygen are also shown to facilitate VEGF mRNA expression in tumor cells. ${ }^{18}$

Serum concentration of VEGF has been investigated in numerous human malignancies, including melanoma, non-Hodgkin's lymphoma, renal cell carcinoma, brain, colorectal, small cell lung, and vulvar carcinoma. ${ }^{6,7,8,14,19}$ In these studies, elevated serum VEGF correlated with poor prognosis. To date, there exists no prospective study evaluating the usefulness of s-VEGF as a prog-

Table 3. Cox proportional hazards multivariable regression of parameters potentially influencing overall survival in advanced laryngeal cancer patients.

\begin{tabular}{lcc}
\hline Variable & Risk ratio & $p$ Value \\
\hline Serum VEGF & 1.474 & .065 \\
Treatment & 0.895 & .574 \\
Stage & 1.285 & .253 \\
Site & 1.408 & .125 \\
Age & 1.027 & .102 \\
T stage & 1.059 & .733 \\
Growth pattern & 1.441 & .018 \\
\hline
\end{tabular}

nostic factor in human squamous cell carcinoma of the head and neck. Qian et $\mathrm{al}^{11}$ have evaluated serum VEGF levels in nasopharyngeal carcinoma. In their study, they analyzed s-VEGF levels in normal controls, patients with nonmetastatic nasopharyngeal carcinoma, and patients with metastatic nasopharyngeal carcinoma. What they found was that s-VEGF levels $>900 \mathrm{pg} / \mathrm{mL}$ indicated metastasis with a high specificity $(95.4 \%)$ and a low sensitivity (31.8\%). The weakness of this study, as stated by the authors, was that it was not a prospective study and that cor-

Table 4. Cox proportional hazards multivariable regression of parameters potentially influencing overall survival in advanced laryngeal cancer patients treated by surgery.

\begin{tabular}{lcc}
\hline Variable & Risk ratio & $p$ Value \\
\hline Serum VEGF & 1.373 & .310 \\
Stage & 1.189 & .584 \\
Site & 1.432 & .260 \\
Age & 1.036 & .111 \\
T stage & 0.991 & .968 \\
Growth pattern & 1.834 & .012 \\
\hline
\end{tabular}


Table 5. Cox proportional hazards multivariable regression of parameters potentially influencing overall survival in advanced laryngeal cancer patients treated with chemotherapy and radiation therapy.

\begin{tabular}{lcc}
\hline Variable & Risk ratio & $p$ Value \\
\hline Serum VEGF & 1.480 & .199 \\
Stage & 1.427 & .241 \\
Site & 1.373 & .327 \\
Age & 1.014 & .604 \\
T stage & 1.082 & .755 \\
Growth pattern & 1.278 & .228 \\
\hline
\end{tabular}

relation with outcome should be made on pretreatment VEGF levels. This is exactly what our study has investigated. The VA Laryngeal Cooperative Study \#268 provides a unique opportunity to investigate the usefulness of serum VEGF levels, because pretreatment serum is available on the study patients and detailed data exist on the patients' tumors at the time of presentation. Furthermore, very accurate longitudinal follow-up data exist on all the patients included in this study. Altogether, this may allow important conclusions to be drawn concerning pretreatment VEGF levels in advanced laryngeal carcinoma.

The most significant finding in our study is that pretreatment s-VEGF was an independent predictor of overall survival in advanced laryngeal cancer patients. In our multivariate analysis, in which all known risk factors for tumor recurrence were included as covariates, elevated sVEGF levels were associated with decreased overall survival, and serum values greater than the mean imparted a $47 \%$ increased risk of death $(p$ $=.065, \mathrm{RR}=1.47, \mathrm{CI}=0.98-2.23$ ). Although with a two-sided $p$ value it just failed to reach statistical significance, the only factor with a stronger correlation than s-VEGF level was aggressive histologic growth pattern. Despite reaching statistical significance $(p=.0179)$, it imparted only a $44 \%$ increased risk of death $(\mathrm{RR}=$ $1.441,95 \%$ CI $=1.06-1.95)$. Further compelling data in illustrating the importance of s-VEGF were that it was more strongly correlated with ultimate survival than stage, site, T stage, and $\mathrm{N}$ status, which have historically been considered major determinants of overall survival. Similar findings linking increased s-VEGF with poor overall survival in multivariate analyses have been documented in other malignant neoplasms such as breast, lung, ovarian, and brain tumors. ${ }^{20,21}$ In head and neck cancer, Eisma et $\mathrm{al}^{22}$ showed that tissue levels of VEGF and IL-8 cor- related with more aggressive disease, higher TNM stage, more recurrences, and shorter disease-free survival. Similary, Chen et $\mathrm{al}^{23}$ illustrated that elevated s-VEGF and IL-8 correlated with large primary tumor volume. Although these reports point to findings similar to ours, a notable difference is that s-VEGF in our study did not correlate with the stage of disease. This is likely due to the preponderance of T3 tumors in our study, which can vary markedly in tumor volume according to the size of the primary tumor.

When the two arms of the VA Larynx trial were looked at individually using Kaplan-Meier survival curve analysis, elevated s-VEGF was significantly associated with poorer overall survival in the surgical arm $(p=.0018)$ but not in the chemotherapy arm of the trial. Likewise, using Cox regression analysis with s-VEGF as a continuous variable, elevated s-VEGF was significantly associated with poor survival in the surgical arm $(p=.0311)$ but not in the induction chemotherapy group $(p=.1091)$. Knowing that $\mathrm{s}$-VEGF levels correlated with survival in a multivariate analysis of all patients, the question arose as to why the difference existed between the two groups. One possibility is that s-VEGF, as a reflection of tumor angiogenesis, is negated as a predictive factor by the use of the chemotherapeutic agents themselves. In a theory first proposed by Denekamp in $1982,{ }^{24,25}$ rapidly dividing endothelial cells in newly formed tumor blood vessels may render such vessels sensitive to the cytotoxic effects of chemotherapy, much like rapidly dividing cells in hair follicles, bone marrow, and alimentary tract mucosal cells. ${ }^{26}$ This "collateral damage" to the tumor vasculature could contribute to the antitumor efficacy of chemotherapy and make s-VEGF less likely to be a predictive factor for overall survival in the induction chemotherapy group. To support this hypothesis, we performed multivariate analyses in each arm of the VA Larynx trial using the same variables including s-VEGF. We theorized if s-VEGF was not an independent predictor of survival in the chemotherapy arm but was significant in the surgical arm, this would lend some credence to this argument. The multivariate analyses, however, failed to reveal a correlation in either arm with s-VEGF levels. In all likelihood, the most plausible explanation as to why s-VEGF correlated with survival for the entire group of patients but not in each individual arm is strictly related to statistical power. There was a good deal of variability in sVEGF levels throughout the patient population; 
therefore, a larger number of patients was necessary to show correlation with overall survival. This concept that chemotherapy has an antiangiogenic effect remains appealing, however, and merits consideration in future analyses with larger groups of patients.

Another important finding in this study is that $\mathrm{s}$-VEGF levels were markedly elevated in patients with advanced laryngeal carcinoma vs normal healthy controls (319.22 vs $47.83, p<.001)$. Furthermore, the serum VEGF levels between the two arms of the VA Larynx trial (surgery/RT vs induction chemotherapy/RT) were not different from each other as expected (0.941) because of the similar tumor staging in both groups. This raises the possibility that $\mathrm{s}$-VEGF may be a candidate biomarker of tumor presence in head and neck patients. In renal cell carcinoma, Baccala et $\mathrm{al}^{27}$ have shown a decrease in serum VEGF with tumor response to therapy and an increase with recurrence. Studies in nasopharyngeal carcinoma have also shown that s-VEGF may be a biomarker for metastasis. ${ }^{11}$ To further investigate this possibility, however, a prospective trial would need to be performed, with serial s-VEGF levels being drawn and correlated with clinical tumor parameters.

Finally, it is important to note that elevated $\mathrm{s}$-VEGF correlated with a poor performance status $(p=.008)$ in all patients with advanced laryngeal cancer. In patients randomly assigned to the surgical arm of the trial, s-VEGF levels were also elevated in those initially seen with advanced nodal status $(p=.047)$ and supraglottic location of tumor $(p=.022)$. The explanation for all these observations may be that high s-VEGF levels are present in the most aggressive tumors and thus result in greater patient disability.

Several aspects of this study may be perceived as deficiencies and must be expanded on. One of these lies in the fact that platelet counts were not available for the subjects in our study. It has been shown that s-VEGF levels correlate with platelet counts in various human malignancies. ${ }^{28}$ This is a factor in our study that we were unable to control for, because only serum was available for analysis. Other factors such as menstruation and medications that could affect s-VEGF levels were also not taken into account because of lack of data. There were, however, very few women in this study, making menstruation a factor of negligible significance. The use of banked frozen serum for analysis may also be perceived as a deficiency in our study. According to Jacobsen et al, ${ }^{21}$ however, storage time from 2 to 16 years showed no trend or shift on analyzed s-VEGF levels, illustrating the stability of this compound and validating our research design. Finally, it is unfortunate that serial serum samples were not available at various points during the treatment cycles. This would have provided critical information in terms of s-VEGF level changes in response to treatment and its use as a biomarker for laryngeal cancer. We are currently performing such studies in other laryngeal preservation protocols.

\section{CONCLUSIONS}

1. Pretreatment s-VEGF levels in advanced laryngeal carcinoma were an independent predictor of overall survival in the VA Laryngeal Cooperative Study Group Trial \#268.

2. Healthy volunteers had a significantly lower s-VEGF level than advanced laryngeal cancer patients, indicating that s-VEGF may be a valuable serum marker for detecting laryngeal squamous cell carcinoma.

3. Laryngeal cancer patients with lower performance statuses tended to have significantly elevated s-VEGF levels.

4. Elevated serum VEGF levels in the surgical arm of the VA Laryngeal Study correlated with advanced nodal disease, supraglottic location of tumor, and decreased overall survival.

5. Overall, there were many indications in this report of elevated s-VEGF levels correlating with aggressive disease and poor prognosis. Further investigations are warranted to explain the importance of this systemically detected marker of angiogenesis.

\section{REFERENCES}

1. Folkman J. Angiogenesis and cancer, vascular, rheumatoid and other disease. Nat Med 1995;1:27-31.

2. Folkman J. What is the evidence that tumors are angiogenesis dependent? J Natl Cancer Inst 1990;82:4-6.

3. Hanahan D, Folkman J. Patterns and emerging mechanisms of the angiogenic switch during tumorigenesis. Cell 1996;86:353-364.

4. Dvorak HF, Brown LF, Detmar M, Dvorak AM. Vascular permeability factor/vascular endothelial growth factor, microvascular hyperpermeability and angiogenesis. Am J Pathol 1995;146:1029-1039.

5. Fujisaki K, Mitsuyama K, Toyonaga A, Matsuo K, Tanikawa K. Circulating vascular endothelial growth factor in patients with colorectal cancer. Am J Gastroenterol 1998; 93:249-252.

6. Salven P, Ruotsalaine NT, Mattson K, Joennsu H. High pretreatment serum level of vascular endothelial growth 
factor (VEGF) is associated with poor outcome in small cell lung cancer. Int J Cancer 1998;79:144-146.

7. Salven P, Heikkila P, Joennsu H. Enhanced expression of vascular endothelial growth factor in metastatic melanoma. Br J Cancer 1997;76:930-934.

8. Salven P, Teerenhovi L, Joennsu H. A high pretreatment serum vascular endothelial growth factor concentration is associated with poor outcome in non-Hodgkin's lymphoma. Blood 1997;90:3167-3172.

9. Takano S, Yoshii Y, Kondo S, et al. Concentration of vascular endothelial growth factor in the serum and tumor tissue of brain tumor patients. Cancer Res 1996;56: 2185-2190.

10. Vermeulen PB, Dirix LH, Martin M, Lemmens J, Van Oosterom AT. Serum basic fibroblast growth factor and vascular endothelial growth factor in metastatic renal cell carcinoma treated with interferon alfa-2b. J Natl Cancer Inst 1997;89:1316-1317.

11. Qian CN, Zhang CQ, Guo X, et al. Elevation of serum vascular endothelial growth factor in male patients with metastatic nasopharyngeal carcinoma. Cancer 2000;88: 255-261.

12. Wolf GT, Hong WK, Fisher S, et al. Induction chemotherapy plus radiation compared with surgery plus radiation in patients with advanced laryngeal cancer. N Engl J Med 1991;324:1685-1690.

13. Jakobsson PA. Histologic classification in grading of malignancy in carcinoma of the larynx. Acta Radiol 1973;12: $1-8$.

14. Hefler L, Tempfer C, Obermair A, et al. Serum concentrations of vascular endothelial growth factor in vulvar cancer. Clin Cancer Res 1999;5:2806-2809.

15. Neufeld G, Cohen T, Gitay-Goren H, et al. Similarities and differences between the vascular endothelial growth factor (VEGF) splice variants. Cancer Metastasis Rev 1996;15:153-158.

16. Terman B, Dougher-Vermazen M. Biologic properties of VEGF/VPF receptors. Cancer Metastasis Rev 1996;15: 159-163.

17. Brown LF, Berse B, Jackman RW, et al. Increased expression of vascular permeability factor and its receptors in kidney and bladder carcinomas. Am J Pathol 1993;143: $1255-1259$

18. Stein I, Neeman M, Shweiki D, Itin A, Keshet E. Stabilization of vascular endothelial growth factor mRNA by hypoxia and hypoglycemia and coregulation with other ischemia-induced genes. Mol Cell Biol 1995;15: 5363-5368.

19. Kumar H, Heer K, Lee PW, et al. Preoperative serum vascular endothelial growth factor can predict stage in colorectal cancer. Clin Cancer Res 1998;4:1279-1285.

20. Linderholm B, Tavelin B, Grankvist K, Henriksson R. Vascular endothelial growth factor is of high prognostic value in node-negative breast carcinoma. J Clin Oncol 1998;16:3121-3128.

21. Jacobsen J, Rasmuson T, Grankvist K, Ljungberg B. Vascular endothelial growth factor as prognostic factor in renal cell carcinoma. J Urol 2000;163:343-347.

22. Eisma RJ, Spiro JD, Kreutzer DL. Role of angiogenic factors: coexpression of interleukin-8 and vascular endothelial growth factor in patients with head and neck squamous carcinoma. Laryngoscope 1999;109:687-693.

23. Chen Z. Malhotra PS, Thomas GR, et al. Expression of proinflammatory and proangiogenic cytokines in patients with head and neck cancer. Clin Cancer Res 1999;5: 1369-1379.

24. Kerbel RS, Viloria-Petit A, Klement G, Rak J. 'Accidental' anti-angiogenic drugs: anti-oncogene directed signal transduction inhibitors and conventional chemotherapeutic agents as examples. Eur J Cancer 2000;36:1248-1257.

25. Denekamp J. Endothelial cell proliferation as a novel approach to targeting tumour therapy. Br J Cancer 1982;45: 136-139.

26. Kerbel RS. Inhibition of tumor angiogenesis as a strategy to circumvent acquired resistance to anti-cancer therapeutic agents. Bioessays 1991;13:31-36.

27. Baccala AA, Zhong H, Clift SM, et al. Serum vascular endothelial growth factor is a candidate biomarker of metastatic tumor response to ex vivo gene therapy of renal cell cancer. Urology 1998;51:327-332.

28. Verheul HM, Hoekman K, Luykx-de Bakker S, et al. Platelet: transporter of vascular endothelial growth factor. Clin Cancer Res 1997;3:2187-2190. 